\title{
Flying-Hot-Wire Study of Flow Past an NACA 4412 Airfoil at Maximum Lift
}

\author{
Donald Coles* \\ California Institute of Technology, Pasadena, Calif. \\ and \\ Alan J. Wadcock $\dagger$ \\ Beam Engineering, Inc., Sunnyvale, Calif.
}

\begin{abstract}
Hot-wire measurements have been made in the boundary layer, the separated region, and the near wake for flow past an NACA 4412 airfoil at maximum lift. The Reynolds number based on chord was about 1,500,000. Special care was taken to achieve a two-dimensional mean flow. The main instrumentation was a flying hot wire; that is, a hot-wire probe mounted on the end of a rotating arm. The probe velocity was sufficiently high to avoid the usual rectification problem by keeping the relative flow direction always within a range of \pm 30 deg from the probe axis. A digital computer was used to control synchronized sampling of hot-wire data at closely spaced points along the probe arc. Ensembles of data were obtained at several thousand locations in the flowfield. The data include intermittency, two components of mean velocily, and twelve mean values for double, triple, and quadruple products of two velocity fluctuations. No information was obtained about the third (spanwise) velocity component. An unexpected effect of rotor interference was identified and brought under reasonable control. The data are available on punched cards in raw form and also after use of smoothing and interpolation routines to obtain values on a fine rectangular grid aligned with the airfoil chord. The data are displayed in the paper as contour plots.
\end{abstract}

\section{Introduction}

$\mathbf{T}$ HE rate of development of methods for calculating turbulent flows is limited by a scarcity of accurate measurements. The present research is an attempt to meet this need by experimentally describing the trailing-edge separation process on an airfoil operating near maximum lift, together with the rapid relaxation process in the near wake to a distance of about 1 chordlength downstream from the trailing edge.

\section{Previous Work}

The experimental material of the Stanford contest in $1968^{1}$ included several studies of turbulent separation or nearseparation in diffusers and on airfoils. In no case were the measurements carried beyond separation. The main reason was probably that the best instrument available at the time (the conventional hot-wire probe) is an instrument of limited directional response which tends to rectify the velocity signal in regions of intermittently reversed flow. The most complete data, published in 1950 by Schubauer and Klebanoff, ${ }^{2}$ have been studied by many researchers and found to be unsatisfactory in several respects. The mean flow was definitely three-dimensional in the region of rising pressure, and the measured values for the turbulent stresses are almost certainly too large, perhaps by $30 \%$.

In recent years, a new instrument which avoids the rectification problem has become generally available. This is the laser-Doppler velocimeter (LDV) with frequency of fset. This

Received June 19, 1978; presented as Paper 78-1196 at the AIAA 11 th Fluid and Plasma Dynamics Conference, Scattle, Wash., July 10 . 12, 1978; revision received November 9, 1978. Copyright (c) American Institute of Aeronautics and Astronautics, Inc., 1978. All rights reserved.

Index categories: Aerodynamics; Boundary Layers and Convective Heat Transfer-Turbulent; Viscous Nonboundary-Layer Flows.

"Professor of Aeronautics. Fellow AIAA.

†Research Scientist; formerly Graduate Student, California Institute of Technology. instrument has not yet been fully exploited, perhaps because it has its own problems in regions of high turbulence level. The most important of these is statistical bias in lightly seeded or unseeded flows. The LDV has recently been used, for example, by Simpson et al. ${ }^{3}$ to study separating flow in a diffuser. However, the measurements are not sufficiently detailed to allow accurate modeling of mixing processes in the separated region.

The nearest equivalent to the experiment reported here is the recent work by Seetharam and Wentz. ${ }^{4}$ Both experiments deal with flow past an airfoil at high angle of incidence at about the same Mach number and Reynolds number. Both experiments include data in the wake to about 1 chordlength downstream from the trailing edgc. Both airfoils had an aspect ratio of about 2 and were mounted between plane parallel sidewalls in a compound test section. However, the two experiments are completely different in attack and execution. Seetharam and Wentz used only pressure instrumentation of various types, and it is difficult to estimate the magnitude of any errors which may be present in their data.

\section{The Flying Hot Wire}

The main experimental technique of the present research uses a flying hot wire mounted on the end of a whirling arm. The rectification problem is thus avoided by biasing the relative velocity. In practice, the tip speed of the whirling arm can be made large enough so that the direction of the relative flow at the probe (a standard commercial $X$-array) is always within the useful range of about $\pm 30 \mathrm{deg}$ with respect to the probe axis.

The flying-hot-wire technique has several special features which are discussed at length in a report on instrumentation by Coles et al. ${ }^{5}$ (this report will be referred to as $\mathrm{CCW}$ ) and in the thesis by Wadcock ${ }^{6}$ and the contract report by Coles and Wadcock ${ }^{7}$ which are parent to the present technical paper (the latter report will be referred to as $\mathrm{CW}$ ). One special feature (good) is that data are obtained along a line rather than at a point. Another (bad) is that the wake of the whirling arm is a 
substantial moving disturbance in the flow. A third (good) is that the hot-wire probes are inherently self-calibrating in pitch. A fourth (bad) is that hot-wire signals must be transmitted through slip rings. A fifth (bad) is that the relative position of probe and model is not easy to measure. A sixth (good and bad) is that the technique is necessarily digital. The probe signals are not statistically stationary; the concept of spectrum, for example, plays no role at all in the measurements. Mean values are, by definition, ensemble mean values obtained by repeated sampling at fixed points in the flow.

The first two of these special features are probably the most important in practice. That the moving probe is a line instrument is important because one issue in any measurement of turbulence is the time required to obtain a stable mean value. In our application, a 5-min run yielded an ensemble of thousands of velocity samples from each of two probes at each of 60 or more points along an arc in the flow. At each of the 60 points, moreover, the flow was observed over the full 5 min. The benefits of a stable mean are conspicuous in the final results of this research. We know of no other experiment of comparable complexity in which the experimental data can be differentiated with any confidence.

The second important feature, which was viewed from the outset as a calculated risk, was the possibility that the flyinghot-wire instrumentation might interfere with and change the flow under study. Our original expectation was that the wake of the moving probe might noticeably affect the momentum distribution along its trajectory. What happened was quite different. Because the separation line is not fixed by the geometry, flow separation from an airfoil is exquisitely sensitive to small changes in the ambient flow at large distances. Such changes did occur. Blockage of the freestream by the rotor hub caused a measurable dependence of the flow in the boundary layer and wake on the position of the rotor in the test section. Considerable time was required to understand this effect and minimize its importance.

Our conclusion at the end is that the flying hot wire is a difficult but usable instrument. It is probably best suited to bluff-body flows, where close approach to a surface is not mandatory and where separation is fixed or nearly fixed by the geometry of the model. When the same flying-hot-wire instrumentation was used by Cantwell $^{8}$ to study vortex shedding from a circular cylinder, no measurable interference effects were found.

\section{Model and Instrumentation}

In principle, one airfoil section would serve as well as another to meet the primary objectives of the present research. What was wanted was a well-documented section known to have good stalling characteristics; i.e., a smooth variation of lift with angle of attack near maximum lift, with gradual trailing-edge separation. The section chosen was the NACA 4412 , mainly because the aerodynamic properties of this section have been measured in considerable detail by Pinkerton ${ }^{9}$ for Reynolds numbers from 100,000 to $8,200,000$.

\section{Airfoil Model}

The airfoil model was fabricated of fiberglass-reinforced polyester resin in a mold of polished sheet metal. The chord $c$ was $90.12 \mathrm{~cm}$ and the span was $199.1 \mathrm{~cm}$. The model was made in two pieces - a main body and a full-span hatch cover. The hatch extended from $x / c=0.18$ to $x / c=0.73$ on the pressure surface and gave access to the interior for installation of ribs, spars, and pressure instrumentation.

Numerous pressure orifices of $0.8-\mathrm{mm}$ diam were drilled in the model. The NACA 4412 section has a finite trailing-edge thickness, and several pressure orifices were installed actually in the trailing edge. Two pressure-scanning valves driven by 12 -step rotary solenoids were mounted inside the wing. Altogether, 89 pressure orifices could be connected at one time. Most of these were at midspan, but a number were at one-quarter and three-quarter span to observe the degree of two-dimensionality of the flow.

The wing was mounted horizontally between vertical false walls in the cylindrical test section of the GALCIT (Graduate Aeronautical Laboratories, California Institute of Technology) 10-ft wind tunnel. The wing had to be mounted upside down (i.e., lifting downward) because of the peculiar character of the flying-hot-wire instrumentation. The geometry of the false walls and the location of the wing in the test section are described in detail in $\mathrm{CW}$. No unusual procedures were used to prepare the wind tunnel for the airfoil experiment, and no screens or other devices were used to improve the quality of the freestream flow.

\section{Traverse, Strut, and Rotor}

Figure 1 is an outline drawing of the flying-hot-wire apparatus and the airfoil model in the GALCIT tunnel. The horizontal traverse below the tunnel was a salvaged lathe bed and carriage. The vertical traverse was a milling-machine slide. The rotor was supported from the vertical traverse by a strut of heavy rectangular steel tubing. The part of this strut exposed to the airflow was streamlined by wooden cladding shaped to an NACA 0024 profile section. The test section of the wind tunnel has a narrow full-length slot at the bottom which allowed traversing in the streamwise direction. The slot was sealed by a cloth strip with two zipper closures running upstream and downstream from the strut.

Mounted in the strut was a flat printed-circuit motor. The flying-hot-wire rotor was attached directly to the shaft of this motor. Rotor speed was accurately controlled by a phaselocked-loop (PLL) servosystem which was designed by $B$. Cantwell and is described in more detail in CCW. The angular position of the motor shaft was encoded by a 256-tooth precision gear and a magnetic pickup which produced a clean TTL pulse train called the ENCODER signal. This signal served as feedback input to the PLL phase detector, whose other input originated in a $200-\mathrm{kHz}$ crystal-controlled pulse train. A counter, controlled by thumbwheel switches, divided this $200-\mathrm{kHz}$ frequency by any desired decimal integer from 1 to 999 . The PLL servosystem responded smoothly to commands to change speed and could maintain lock in spite of large variations of torque on the rotor within each revolution.

\section{Velocity and Pressure Instrumentation}

Constant-temperature anemometer circuits were constructed particularly for the present experiments according to a design recommended by Perry and Morrison. ${ }^{10}$ The main addition to the design was the incorporation of a hybrid intermittency circuit in each channel. The hot-wire probes were commercial X-arrays whose sensing elements were platinum-plated tungsten wires $5 \mu \mathrm{m}$ in diameter and $1 \mathrm{~mm}$ long. The operating resistance ratio was normally about 1.41.5. Hot-wire signals were carried by shielded cables through the hollow rotor arms to the hub, through the hollow motor shaft to mercury slip rings, and through the hollow strut to the hot-wire equipment below the tunnel.

An electrical signal representing tunnel temperature was obtained from an electronic thermometer. Pressures were measured using two electronic differential manometers. One manometer was permanently connected to the two sides of a pitot-static tube mounted from the tunnel roof to measure a reference dynamic pressure $Q_{\text {ref }}=\rho\left(q_{\mathrm{ref}}\right)^{2} / 2=p_{\mathrm{tref}}-p_{\text {sref }}$. The second differential manometer was connected to the pressure-scanning system inside the wing to measure model surface pressure $p_{m}-p_{t_{\text {ref }}}$. One input to the pressurescanning system was $p_{s_{\text {rel }}}$ from the static side of the roofmounted pitot-static tube. The two manometers could, therefore, be checked against each other by selecting the proper valve port. Agreement in $Q_{\text {ref }}$ was always within one or two parts in a thousand. 


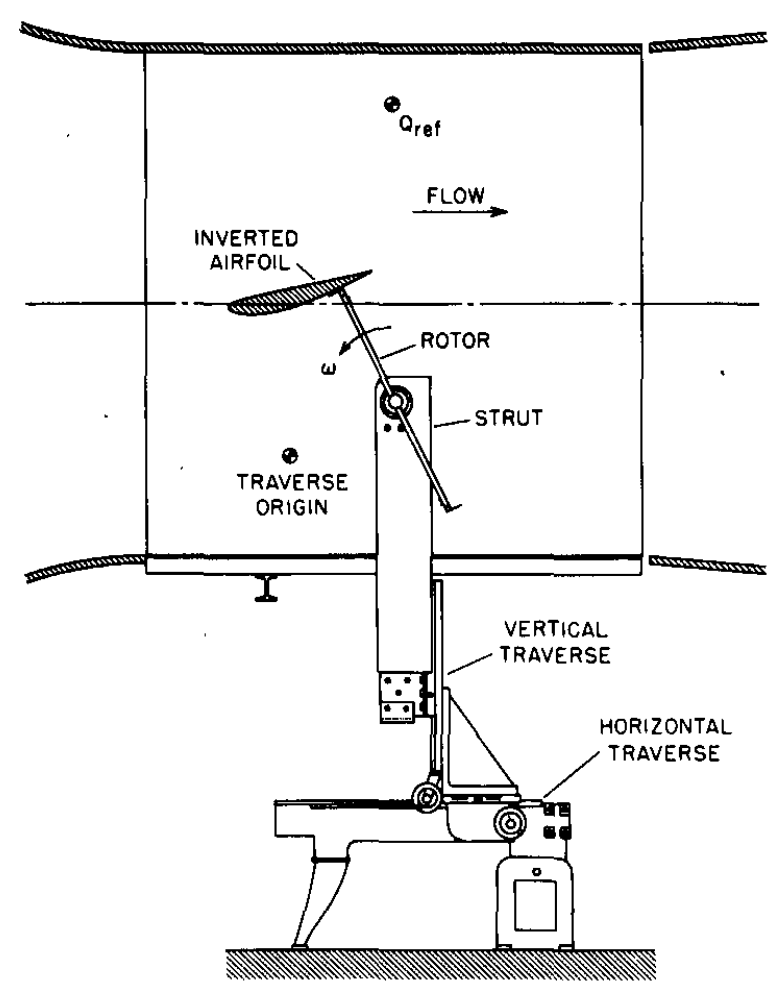

Fig. 1 Side view of flying-hot-wire apparatus and traverse in test section of GALCIT 10-ft wind tunnel.

\section{Data Acquisition}

Data from the experiment were recorded by a computercontrolled data acquisition system. Control was shared by data-logic circuitry in the flying-arm controller, control-logic circuitry in the analog-to-digital converter (ADC), and directmemory-access circuitry in the computer. The function of the flying-arm controller, as described in detail in CCW, was to arrange for a burst or frame of 12 words, spaced at intervals of $5 \mu \mathrm{s}$, to be acquired each time a geartooth passed the magnetic pickup. Thus, the ENCODER signal controlled not only the rotor speed but also the timing, and hence the position in the flow, of the hot-wire measurements. The rotor diameter was $151.4 \mathrm{~cm}$. With 256 equally spaced sampling positions, digital data were obtained every $1.86 \mathrm{~cm}$ along the probe arc. The computer was instructed to verify at the end of each revolution that the data had the correct format and were properly synchronized with rotor position. During the remaining time, the computer was free to edit and dispose of data obtained during earlier revolutions.

Eight of the twelve analog data channels were miscellaneous signals (e.g., tunnel temperature, tunnel dynamic pressure, model surface pressure, scanning valve position). Data from these eight channels were recorded only as mean values over each revolution of the rotor.

Four data channels were reserved for hot-wire data from two X-arrays, one on each arm of the rotor. During each halfrevolution, the computer saved verbatim the data from the $\mathrm{X}$ array which was currently advancing into the stream and discarded the data from the X-array which was currently retreating. There were, therefore, two independent observations of the flow over the same 180-deg arc during each revolution.

The rotor speed during the hot-wire measurements was 375 $\mathrm{rpm}$. The tip speed was $29.73 \mathrm{~m} / \mathrm{s}$. The ENCODER frequency was $200,000 / 125=1600 \mathrm{~Hz}$. During a run or file, the ADC rate was 200,000 words per second (wps), intermittent. The mean transfer rate to core was 19,200 wps. The mean transfer rate to magnetic tape was 3200 wps. Each data file lasted for 2048 revolutions. There are 85 such files in the main data base, representing $89,128,960$ words of data on tape and about $7.74 \mathrm{~h}$ of tunnel time (about $13 \mathrm{~h}$ of elapsed time). In this mass of data, one word is known to have been incorrectly recorded, and one tape record out of 43,520 was unreadable.

\section{Flow Control}

The strategy of the research called for extensive hot-wire measurements at only one angle of incidence and only one Reynolds number. The values finally selected were $\alpha=13.87$ deg (nominally $14 \mathrm{deg}$ ) and $R e=q_{\mathrm{ref}} \mathrm{c} / \nu=1,520,000$. The average tunnel temperature was $24.3^{\circ} \mathrm{C}$ and the barometric pressure was $72.9 \mathrm{~cm} \mathrm{Hg}$. The reference dynamic pressure $Q_{\text {ref }}$ was $3.13 \mathrm{~mm} \mathrm{Hg}$ (nominally $10 \mathrm{lbf} / \mathrm{ft}^{2}$ ). The density $\rho$, kinematic viscosity $\nu$, and reference velocity $q_{\text {rer }}$ were 0.00114 $\mathrm{gm} / \mathrm{cc}, 0.161 \mathrm{~cm}^{2} / \mathrm{s}$, and $27.13 \mathrm{~m} / \mathrm{s}$, respectively.

\section{Uncontrolled Flow}

Boundary-layer trips (of tape trimmed to have a sawtooth leading edge) were at tached to both surfaces of the airfoil. On the suction side, the trip was centered at $x / c=0.025$; on the pressure side, the trip was centered at $x / c=0.103$. No evidence of a leading-edge separation bubble was found at the Reynolds number of the experiment.

The surface-pressure distribution initially measured on the airfoil at $14 \mathrm{deg}$ angle of incidence, with no flow control, is shown in the left part of Fig. 2. The pressure has been made dimensionless as $C_{p}=\left(p_{m}-p_{s_{\mathrm{ref}}}\right) / Q_{\mathrm{ref}}$. Separation was found to occur symmetrically but nonuniformly, being considerably further forward at one-quarter and three-quarter span than at midspan. This degree of three-dimensionality was judged to be unacceptable. The dimensionless total pressure $H / Q_{\text {ref }}=\left(p_{t}-p_{\text {atm }}\right) / Q_{\text {rel }}$, measured in the wake under essentially the same conditions, is shown in the left part of Fig. 3. These data were obtained from three vertical rakes which were mounted $115 \mathrm{~cm}$ downstream from the trailing edge in line with the three rows of pressure orifices on the airfoil at one-quarter, one-half, and three-quarter span. The degree of three-dimensionality is again unacceptable.

\section{Controlled Flow}

Considerable effort was spent, as described in $\mathrm{CW}$, in the application of different methods of active flow control at the sidewalls in the vicinity of the separated region. These methods included area suction and slot blowing. There was no substantial improvement in the flow. Success was finally achieved by a simple passive method, using sheet-metal flow guides attached to the sidewalls, as shown in Fig. 4. The guides were adjusted until an acceptable flow was obtained in the wake, as demonstrated at the right in Fig. 3. A corresponding improvement was found in the pressure distribution on the airfoil, as shown at the right in Fig. 2. It is remarkable that there was almost no change in the flow at midspan, either near the airfoil or in the wake, in spite of large changes taking place in the flow near the sidewalls.

With the flow guides left fixed in their optimum position for $14 \mathrm{deg}$ angle of incidence, the airfoil was pitched through the range from $-4 \mathrm{deg}$ to $16 \mathrm{deg}$. The wake profiles were found to remain essentially two-dimensional. Surface pressure distributions measured at the same time are tabulated in $\mathrm{CW}$. Lift coefficients, obtained by integrating these pressure distributions, are compared in Fig. 5 with Pinkerton's data, ${ }^{9}$ which refer to flow at midspan for a finite wing of aspect ratio 6. Pinkerton corrected his angles of incidence by estimating (according to the figure, underestimating) the downwash velocity at midspan.

\section{Geometry}

For the hot-wire data to be fully useful, the position of the probe relative to the airfoil had to be known to within a fraction of a millimeter under actual test conditions, with the tunnel running and the probe flying. The necessary 

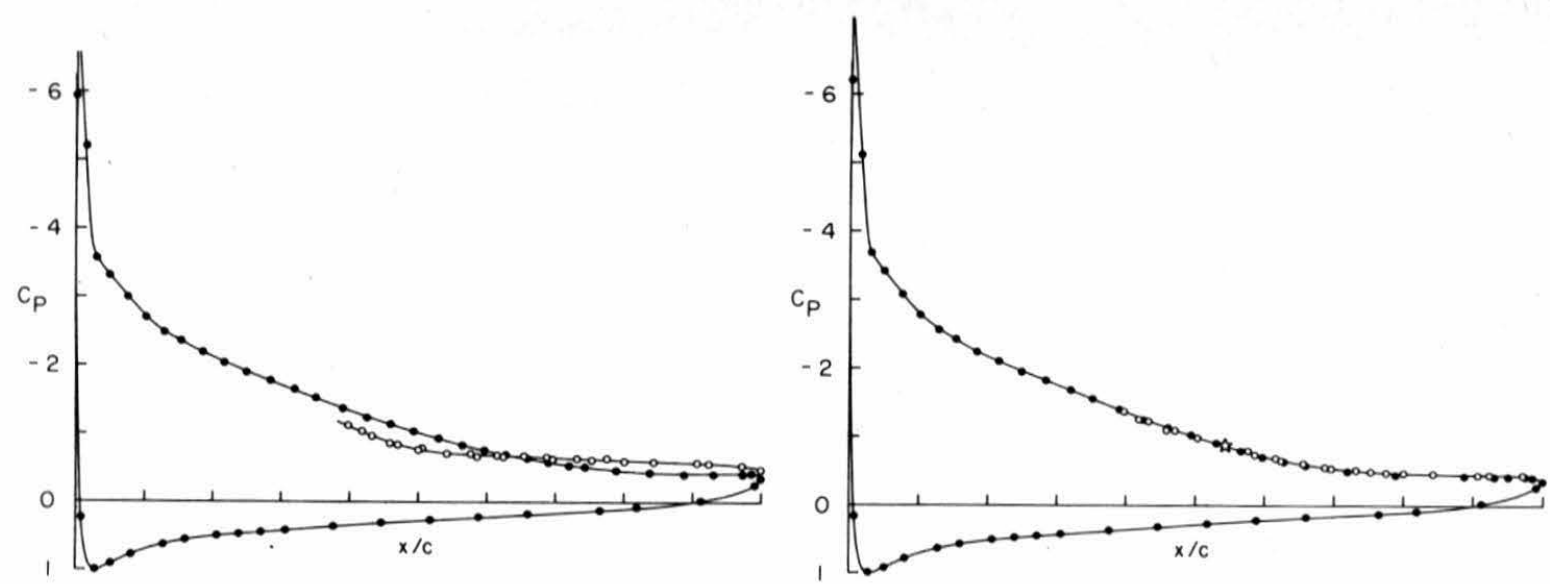

Fig. 2 Left-surface-pressure distribution at 14 deg angle of incidence with no flow control (solid symbols: data at midspan; open symbols: data at one-quarter and three-quarter span). Right-similar data with flow guides in optimum position.
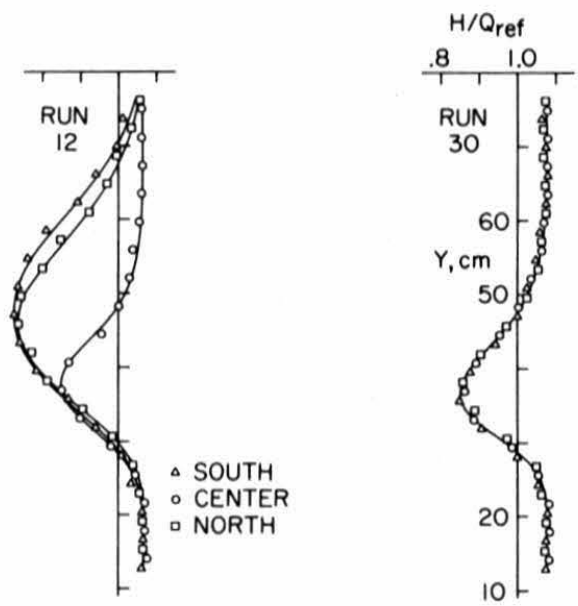

Fig. 3 Left-total-pressure profiles in wake at one-quarter, one-half, and three-quarter span with no flow control. Right-similar data with flow guides in optimum position.

measurements were complicated by the fact that the probe and the airfoil were attached to the building at points several meters apart (cf. Fig. 1).

\section{Model Position}

With the tunnel off, the position of the airfoil with respect to the traverse system was measured by a combination of methods. Data were first obtained by attaching a dial test indicator to one end of the rotor and traversing vertically at various points along the chord (while oscillating the rotor by hand) until the indicator reading was zero. This process determined part of the airfoil surface as the envelope of a series of circles of known center but unknown radius. The radius in question was established to an accuracy of $0.01 \mathrm{~cm}$ or better by observing one of the hot-wire probes and its close reflection in the model surface, the probe radius having previously been measured independently using a cathertometer.

The deflection of the airfoil under air load was measured with the aid of a small optical proximity sensor mounted on one arm of the rotor. The sensor consisted of a light-emitting diode and a phototransistor which illuminated and viewed along lines intersecting a few millimeters from the face of the detector. To obtain a strong sensor signal, a strip of goldcoated reflective tape was attached to the wing surface in the plane of rotation of the rotor, about $11 \mathrm{~cm}$ to one side of the midspan pressure orifices. A peak-reading circuit, reset at the beginning of each revolution, was used to hold the sensor

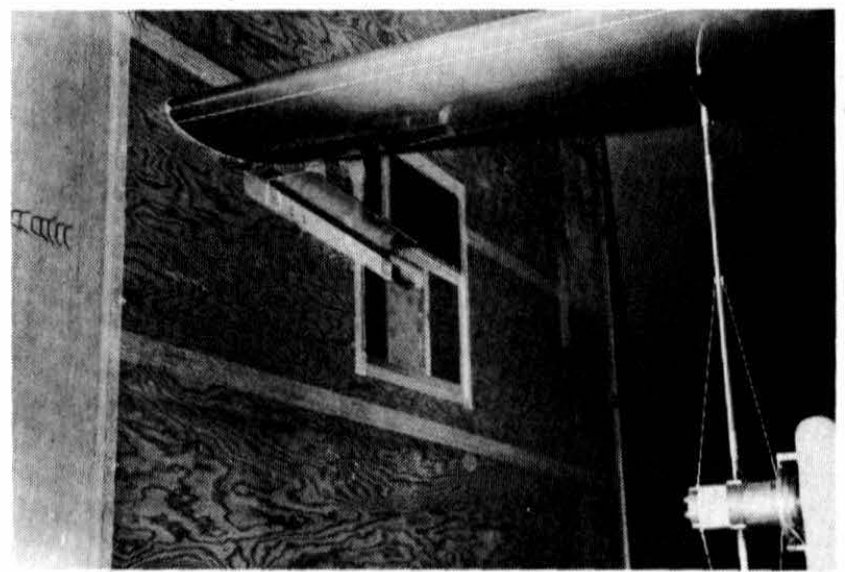

Fig. 4 View of sheet-metal flow guide in final position on sidewall. Upper rectangle on wall behind flow guide is plenum chamber for flow control; lower rectangle is window.

signal for observation. With the rotor turning at standard speed, the change in sensor signal from the air-off to the airon condition showed that the model displacement could be taken as a simple translation of $0.155 \mathrm{~cm}$ normal to the chord line. This displacement may have been partly model deflection and partly strut deflection, but the distinction is immaterial.

The airfoil position was finally determined by fitting the observed air-on location of the rear part of the suction surface to analytical formulas " which describe the 4412 profile. This process fixed the position of the airfoil chord line and also defined the real angle of incidence to be $13.87 \mathrm{deg}$. A more detailed description of these delicate measurements can be found in $\mathrm{CW}$.

\section{Probe Position}

Finding the position of the sampled data was a separate problem whose solution involved a second application of the optical proximity sensor. The rotor was first leveled in the horizontal position, using a sensitive spirit level, and clamped. The rotor was next traversed vertically past a narrow reflecting target, and a convenient trigger point was found. The rotor was then set into motion at standard speed $(375$ $\mathrm{rpm})$. The time interval from the trigger point to the first frame of hot-wire data was measured as $684 \mu \mathrm{s}$, corresponding to $1.54 \mathrm{deg}$ of rotation. To this, the probe offset angle of $5.64 \mathrm{deg}$ (the angle by which the probe led the rotor arm) was added, giving a total angle of $7.18 \mathrm{deg}$ from the horizontal reference to the probe position for the first frame of data. 


\section{Probe Calibration}

One of the most attractive features of the flying hot wire is access to automatic calibration of X-arrays in pitch. From the point of view of a probe rotating in a uniform stream, the relative velocity consists of a steady head-on component $\omega R$ and an unsteady component of fixed magnitude $u$, whose direction rotates at a constant rate. The relative magnitude of the two components may conveniently be specified by a parameter $K=\omega R / u_{\infty}$.

\section{Acceptance Angle}

The parameter $K$ determines the part of the probe arc for which the flow direction relative to a conventional 90-deg Xarray lies within the theoretical acceptance angle of $\pm 45 \mathrm{deg}$ from the probe axis. For very slow or no rotation $(K<1)$, the useful range is $\pm 45 \mathrm{deg}$ on either side of top dead center for the rotor arm. For very fast rotation $(K>1)$, the relative flow direction will always be within the acceptance angle. The boundary observed experimentally for one of our probes, as defined by a shallow minimum in one or the other wire voltage, is shown by the open points in Fig. 6 . The probe angle $\phi$ is measured from top dead center and increases in the direction of probe rotation. The dashed line is a theoretical boundary calculated in $\mathrm{CCW}$. The relative flow angle exceeds $\pm 45 \mathrm{deg}$, and the probe is, therefore, unusable, in the dotshaded region.

\section{Wake Interference}

As the rear rotor arm passes through the horizontal position moving upward, the rear probe encounters the wake left behind by the front rotor arm during the preceding halfrevolution. The experimentally observed boundary for such encounters, as defined by a nonzero value for intermittency, is shown by the solid points in Fig. 6. Wake interference occurs in the slant-shaded region. The dashed line is again a calculated boundary from $\mathrm{CCW}$. The left-half of the figure represents the upper half-revolution for a given probe and is the region for which hot-wire data were recorded.

\section{Calibration and Inversion}

The flying-hot-wire technique must cope with several problems which are not typical of the ordinary art of hot-wire anemometry. One is that the probe calibration arc occupies a considerable fraction of the tunnel test section, and nonuniformities of the calibration flow along this arc may be important. A second is that calibration of wire arrays is required over an expanded range of velocities, with an inevitable loss of wire sensitivity at the upper end of this

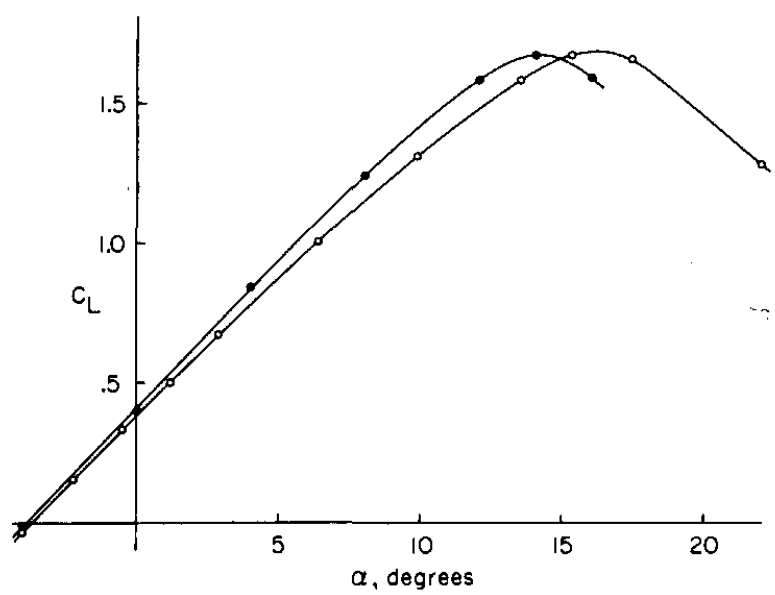

Fig. 5 Section lift coefficient as a function of angle of incidence [solid symbols: present data $\left(R e=1.5 \times 10^{6}\right)$; open symbols: data from Pinkerton (Ref. 9, Table Ilg; effective $R e=6.3 \times 10^{6}$; actual $\left.\left.\operatorname{Re}=2.4 \times 10^{6}\right)\right]$. range. $A$ third is that absolute errors in measured velocity are conserved, and relative errors are, therefore, at least doubled, during conversion from a reference frame fixed in the probe to a reference frame fixed in the tunnel. Finally, a fourth problem is that the technique, being by definition digital, requires the operation of inversion of voltage pairs to obtain velocity vectors to be carried out a very large number of times in a digital computer. The first three problems involve accuracy and are eased by increasing the number of parameters in the formulas representing wire response. The fourth problem involves cost and is eased by decreasing the number of parameters, for the sake of an efficient inversion algorithm. In the present experiments, for example, the inversion operation was carried out nearly thirty million times, requiring altogether about $7 \mathrm{~h}$ of core time in an IBM 370/158 computer. For economy, the wire response was cast in the form of King's law, with the effective cooling velocity taken as the component of relative velocity normal to the wire plus a small additive constant.

\section{Example}

Figure 7 shows the raw material of the posttest calibration for one X-array in terms of the Nusselt numbers $N=e^{2} / \Delta T$, where $e$ is wire voltage and $\Delta T$ is temperature loading ( $N$ is dimensioned, with units $\mathrm{V}^{2} /{ }^{\circ} \mathrm{C}$ ). Probe motion is from right to left along each trajectory. The six symbols in the figure

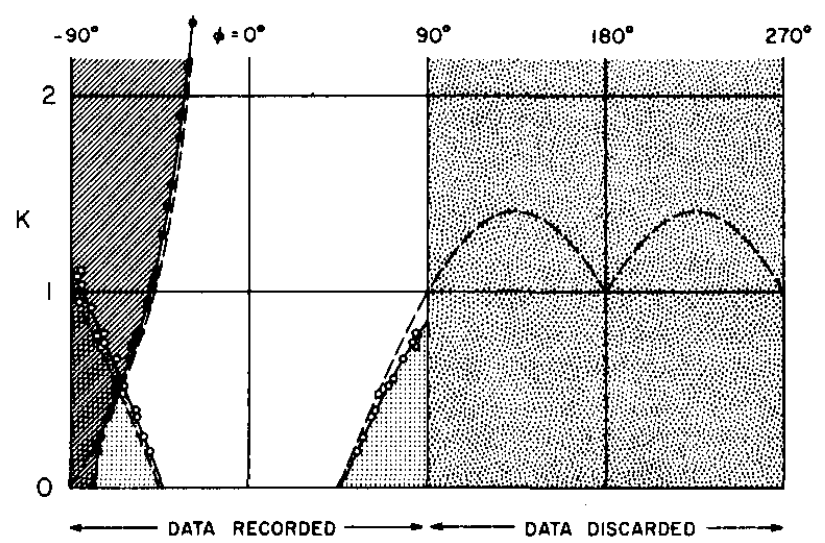

Fig. 6 Experimental wake-interference boundary (solid symbols) and maximum-flow-angle boundary (open symbols) compared to ideal boundaries (dashed lines). Angle $\phi$ is arm position measured from top dead center.

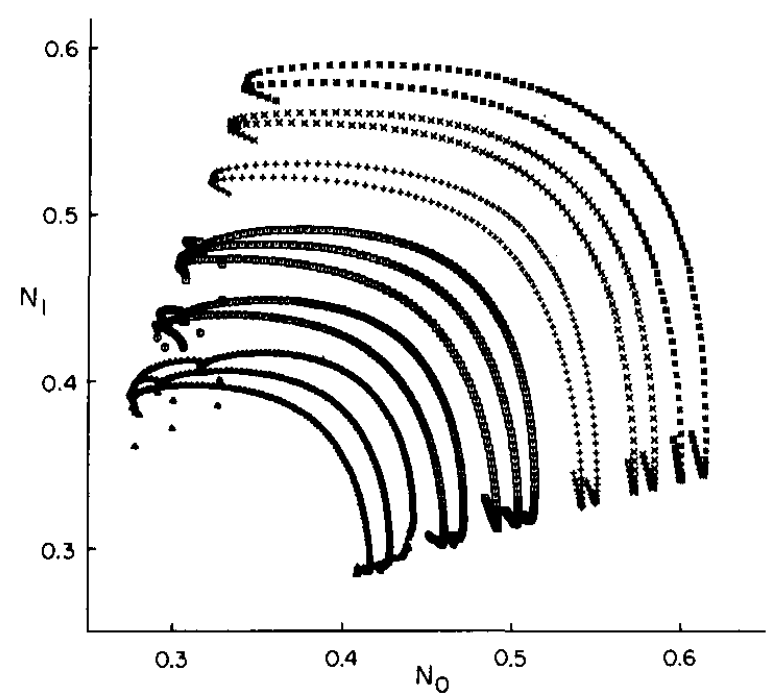

Fig. 7 Raw data from one $X$-array from posttest calibration. Quantity $N=e^{2} / \Delta T$ is dimensioned, with units of $\mathrm{V}^{2} /{ }^{\circ} \mathrm{C}$. 
represent six different tunnel speeds. The two or three trajectories with the same symbol represent different rotor speeds. The data along each trajectory are an average over $\mathbf{2 5 6}$ revolutions and represent from 1-3 min of observation. The entire calibration required a little less than $2 \mathrm{~h}$, mostly spent waiting for the tunnel to reach equilibrium after a change in tunnel speed. The airfoil model was present in the test section, but was pitched to $-4 \mathrm{deg}$, the angle for zero lift. The disturbance at the left in some of the trajectories is caused by passage of the probe through the narrow airfoil wake; this disturbance was outside the region used for probe calibration.

To complete the calibration, the velocity in the calibration flow at each tunnel speed was measured by placing a pitotstatic tube at several points along the probe arc. Details of the formulas used to represent probe response, and the results obtained, can be found in $\mathrm{CW}$ and $\mathrm{CCW}$. After some effort, the calibration-inversion machinery was tuned to a point where the calibration flow in the tunnel reference frame could be reproduced to an accuracy of about $1 \%$ in velocity and 1 deg in flow angle.

\section{Drift}

Inversion of the main bulk of the data was a different matter. Our experience during the present experiments confirms a basic fact of hot-wire anemometry, which is that no two wires ever behave in quite the same way. The wire parameters are subject to continuous and often erratic drift, and also to occasional sudden discontinuities. A hot wire ages. Its surface condition and even its geometry change because of oxidation, thermal distortion, dirt accumulation, and particle impact. The associated electronic circuits also drift, because of sensitivity to changes in ambient temperature or otherwise. The wires used for the main measurements were new and uncooked, and their properties were far from constant during the $21 \mathrm{~h}$ of their use. Considerable time was spent in struggling with this problem of drift. Whatever success was achieved was due to the fact that a particular set of measurements (called a benchmark file) was repeated at frequent intervals throughout the experiment, thus allowing the wire parameters to be adjusted almost continuously during later processing. A detailed account of the struggle with drift is given in $\mathrm{CW}$.

\section{Inversion}

The inversion process for the main experiments used the same algorithms which were earlier applied to calibration data. Each voltage pair yielded a velocity vector relative to the probe. These vectors were treated as a homogeneous population of 2048 samples for each frame and each probe. The individual vectors were not saved. Only the mean vector and mean values for double, triple, and quadruple products of fluctuations were saved. Two passes were made through

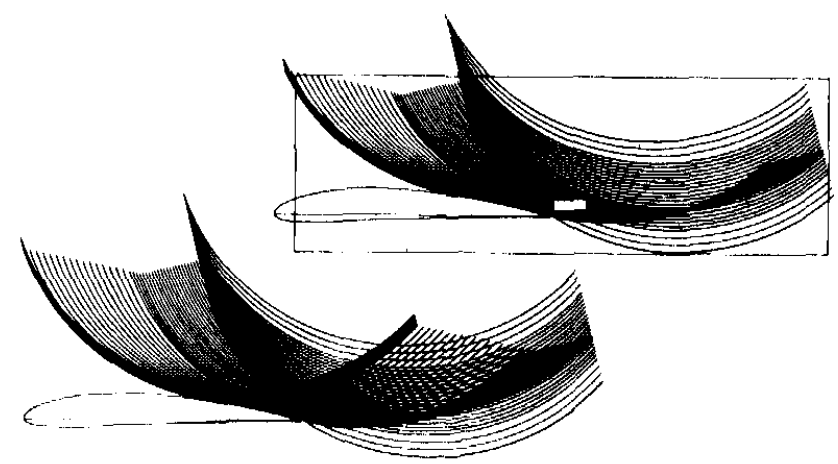

Fig. 8 Lower left-pattern of probe arcs for raw data. Upper right-same pattern after deletion of downstream portion of boundary-layer and grazing traverses. Small rectangle is region involved in area interpolation; large rectangle is region covered by grid. each population - one to obtain the mean vector and one to calculate fluctuations and their mean products. This method substituted intermediate disk storage for the double-precision arithmetic which is usually required when, for example, mean and variance are determined in a single pass. For economy, the two final operations, which were subtraction of the relative velocity due to probe motion and rotation of the resulting absolute velocities into tunnel coordinates, were carried out after ensemble averaging rather than before.

\section{Raw Data}

The results of the inversion process just described are preserved on 14,620 punched cards (85 files, 86 frames, 2 probes), which constitute the raw data of the present experiment. These cards have been filed with the Large-Scale Aerodynamics Branch at NASA Ames Research Center. Each card specifies a particular file, frame, and probe and gives values for the fifteen quantities:

$$
\begin{aligned}
& \langle u\rangle\left\langle u^{\prime} u^{\prime}\right\rangle\left\langle u^{\prime} u^{\prime} u^{\prime}\right\rangle \quad\left\langle u^{\prime} u^{\prime} u^{\prime} u^{\prime}\right\rangle\langle\gamma\rangle \\
& \langle v\rangle\left\langle u^{\prime} v^{\prime}\right\rangle\left\langle u^{\prime} u^{\prime} v^{\prime}\right\rangle \quad\left\langle u^{\prime} u^{\prime} u^{\prime} v^{\prime}\right\rangle \\
& \left\langle v^{\prime} v^{\prime}\right\rangle\left\langle u^{\prime} v^{\prime} v^{\prime}\right\rangle \quad\left\langle u^{\prime} u^{\prime} v^{\prime} v^{\prime}\right\rangle \\
& \left\langle v^{\prime} v^{\prime} v^{\prime}\right\rangle \quad\left\langle u^{\prime} v^{\prime} v^{\prime} v^{\prime}\right\rangle \\
& \left\langle v^{\prime} v^{\prime} v^{\prime} v\right.
\end{aligned}
$$

A tabular description of the card format can be found in $\mathrm{CW}$, together with formulas for calculating the probe position with respect to the airfoil.

\section{Rotor Interference}

The main experiment consisted of four traversesboundary-layer, grazing, horizontal, and vertical. These traverses generated the four families of arcs shown at the lower left in Fig. 8. The arcs run from frame 35 to frame 120, inclusive, and mark the region covered by the raw data of the experiment.

\section{The Problem}

At an early stage of data processing, it became apparent from an inspection of intermittency and other variables that there was a serious problem with probe interference. Our original expectation was that passage of the rotor arm through the fluid would add a local increment of momentum in the direction of probe motion, but that the effect should be small because the affected fluid would move a substantial distance (roughly twice the chord length in the case of the freestream) between successive probe passages. There is strong evidence that the effect just described was small, as expected, and that a different kind of interference was acting. Although the obstacle presented to the flow by the rotor hub was physically small, the drag was an appreciable fraction of the drag of the airfoil itself, and the blockage was substantial. As the traverse and rotor moved forward, particularly during the grazing traverse, the adverse pressure gradient outside the boundary layer was slightly relieved by the acceleration due to blockage, the separation line moved toward the trailing edge, and the wake thickness decreased noticeably. These effects are discussed quantitatively in $\mathrm{CW}$, where direct evidence is cited to support our conclusion that the effect depended only on traverse position and not on probe motion. The evidence includes tabulated surface-pressure data obtained during the hot-wire measurements. These data provide a record of the continuously changing pressure distribution on the airfoil as the traverse and rotor moved in the chordwise direction.

\section{A Protocol}

Fortunately, most of the hot-wire data were obtained with the traverse sufficiently far downstream so that the flow was, 
for practical purposes, independent of rotor position and hence interference-free. In particular, data from the whole of the horizontal and vertical traverses are in good agreement in their common region, which includes most of the separation bubble (cf. the example in Fig. 9).

A convenient division line for the data is the last grazing or first horizontal arc. In preparing for further data processing, the scheme which we used to cope with the interference problem was to discard the part of each boundary-layer or grazing arc downstream of this division line. The truncated data set is shown at the upper right in Fig. 8. To remove small but detectable effects of probe wake interference near frame 35 and of large relative flow angle near frame 120, the arcs have also been shortened to run from frame 40 to frame 115 , inclusive.

\section{Processed Data}

The raw data of the present experiment are defined at points which are closely spaced from the viewpoint of an experimenter, but which might be considered sparse and awkwardly placed from the viewpoint of a numerical analyst. We have, therefore, carried out some further processing to serve the needs of users whose interest is primarily in turbulence modeling. One objective was to use interpolation to redefine the data on a rectangular grid which is sufficiently fine to satisfy the analyst without driving the data beyond their real accuracy. Mostly for convenience in describing the airfoil surface, the grid in question is aligned with the chord line of the airfoil. Another objective was to lay a foundation for calculation of spatial derivatives.

Preparation

The starting point for the interpolation was the information on punched cards, characterized above as raw data. To bypass the rotor interference problem just discussed, the raw data were first reduced to the partial set depicted at the upper right in Fig. 8. A small correction was made to the raw data to force agreement between the hot-wire values for velocity and values obtained from a pitot-static probe which had been traversed through the flow outside the boundary layer and wake on both sides of the airfoil. This correction is discussed in $\mathrm{CW}$; it amounted to an increase of $2 \%$ in the magnitude of the velocity relative to the probe, before conversion to the tunnel reference frame. The correction is not essential for proper interpretation or use of the present data, except perhaps from an aesthetic standpoint.

Each variable was then averaged for the two probes for each frame (except intermittency, which was only observed by one of the probes), and the average value was assigned to the position calculated using the mean probe radius. All velocity components were rotated into airfoil coordinates. Duplicate files, including benchmark files, were represented by a single average file.

The rectangular grid used for processed data had a mesh size of $1 \mathrm{~cm}$ in the chordwise direction and $0.2 \mathrm{~cm}$ in the crossflow direction. The range of the grid was $I X=1(1) 175$ and $I Y=1(1) 296$. The outline of the corresponding rectangle, $174 \times 59 \mathrm{~cm}$, is shown superposed on the airfoil and on the probe arcs in Fig. 8.

\section{Execution}

Each of the fifteen experimental variables was treated separately. In what follows, the symbol $z(x, y)$ can refer to any one of these variables. A small rectangle having its long dimension oriented parallel to the chord line (i.e., parallel to the general direction of the boundary layer and wake) was centered on each of the thousand of data points in succession. The rectangle finally used was $10 \times 3 \mathrm{~cm}$, the $3-\mathrm{cm}$ dimension being chosen so that three data arcs would be involved in the downstream part of the central wake region, where the traverse displacement between arcs was usually $1.27 \mathrm{~cm}$. This rectangle is shown to scale as the white area near the trailing edge of the airfoil in Fig. 8. Other data points were then sought within the small rectangle such that the value of the dependent variable $z$ differed from the value at the centerpoint by no more than a specified small increment. A straight line was drawn connecting each such pair of points, and linear interpolation was used to derive a value for $z$ at any grid lines $I X=$ const which lay between. Short line segments were thus found, of length $5 \mathrm{~cm}$ or less, which lay close to a level curve of the surface $z(x, y)$.

The result of this area-interpolation process was a large array of data along each grid line $I X=$ const. This array was thinned so that no more than two interpolated data points remained in any $1-\mathrm{mm}$ interval in $y$. The two surviving points were those for which the original end values of $z$ were closest to each other. At the end of this process, the number of data points along any grid line was increased by roughly an order of magnitude over the number obtainable by linear interpolation along arcs in the raw data.

Finally, a fitting and smoothing operation was carried out over an interval of $2 \mathrm{~cm}$ in $y$ centered on each grid intersection. Near the edges of the experimentally defined part of the flow, the interval was symmetrically enlarged, as necessary, until at least 12 data points were included. During this operation, the presence of the airfoil was ignored; that is, boundary conditions on $u, v$, and $v / u$ at the airfoil surface were not enforced. The fitted curve was a cubic. Only the value of $z$ at each grid point was saved.

\section{Final Processed Data}

The result of these procedures is illustrated in Fig. 9 for a section through the wake just downstream from the trailing edge. The open symbols were obtained by linear interpolation along arcs in raw data from the horizontal and vertical traverses. The crosses are the result of area interpolation and thinning. The solid line is the fitted curve.

As further examples of final processed data, contour lines of constant chordwise velocity $\langle u\rangle / q_{\text {ref }}$ and constant intermittency factor $\langle\gamma\rangle$ are shown in Fig. 10. Figure 11 is a corresponding set of contour lines for the three dimensionless double correlations or Reynolds stresses. Shading has been used to emphasize peaks and valleys. Note that these figures are based on velocity components resolved along and normal to the chord line. Quite different values and different contours might be obtained near the airfoil if the components were resolved more nearly along and normal to the airfoil surface. Our main conclusion from the Reynolds-stress data is that the separation process is relatively regular up to the trailing edge of the airfoil. The real challenge to un-

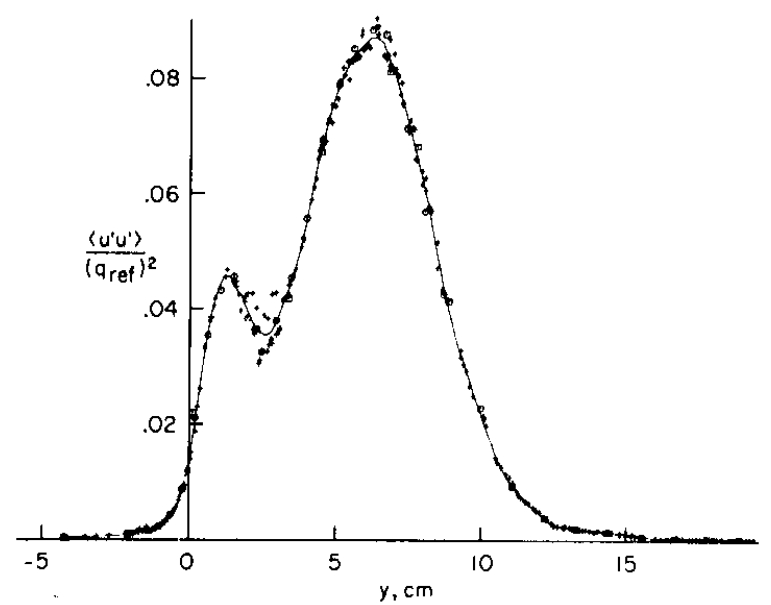

Fig. 9 Typical data for $\left\langle u^{\prime} u^{\prime}\right\rangle /\left(q_{\text {ref }}\right)^{2}$ at $x=98.86 \mathrm{~cm}(I X=93)$. Open symbols: linear interpolation along probe ares in raw data; crosses: area interpolation after thinning 10 a maximum of two points/mm in $y$; solid line: fitted curve. 

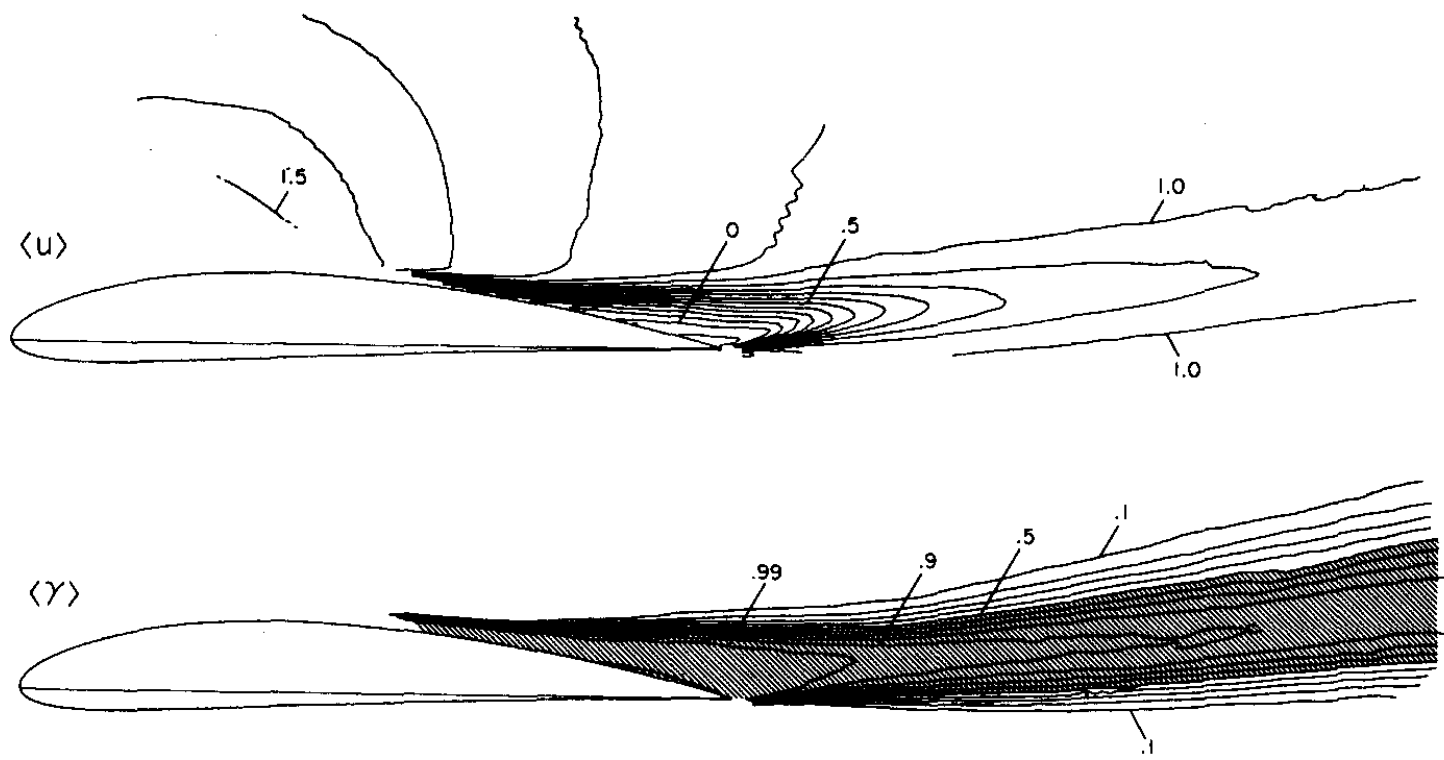

Fig. 10 Contour plots of chordwise mean velocity $\langle u\rangle / q_{\text {ref }}$ and intermittency factor $\langle\gamma\rangle$. Contour intervals is 0.1 .
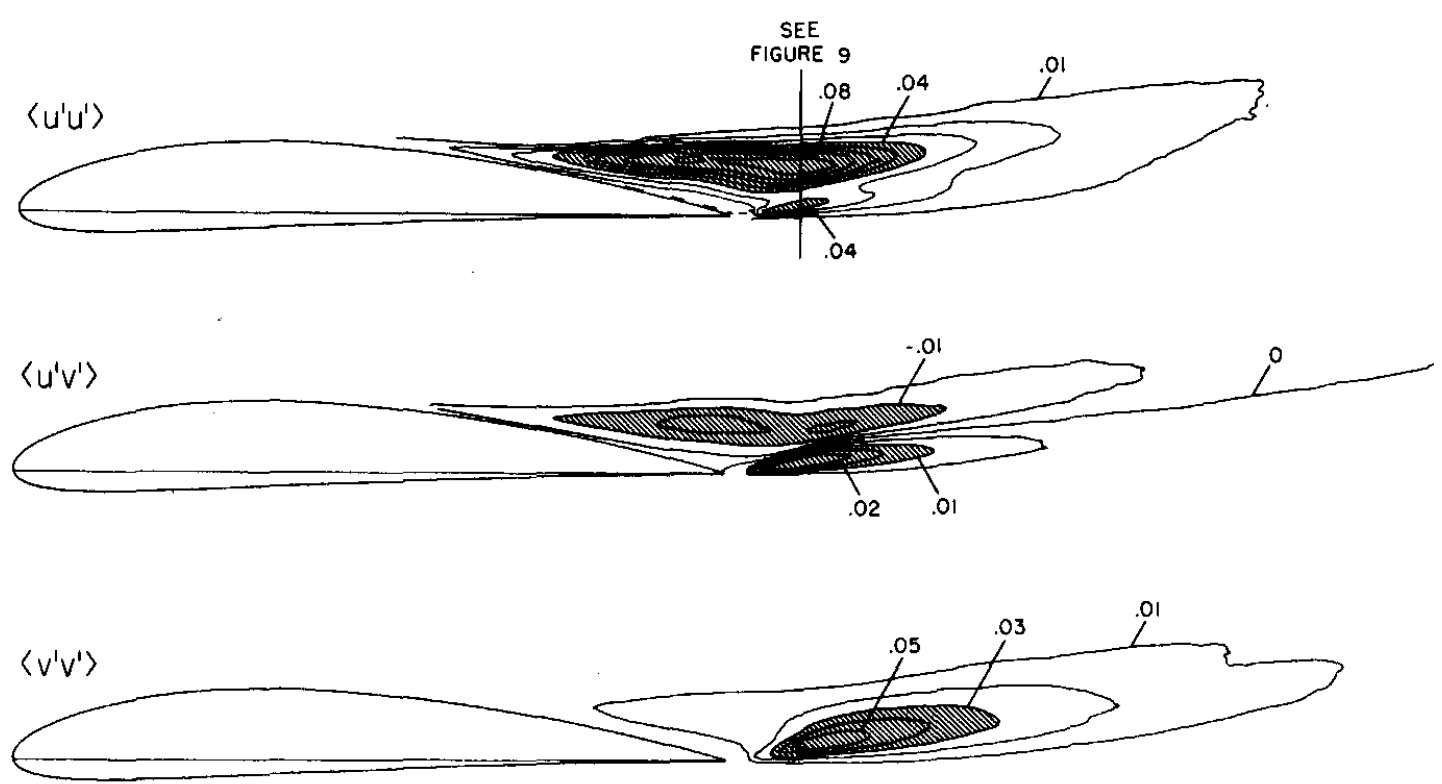

Fig. 11 Contour plots of double correlations $\left\langle u^{\prime} u^{\prime}\right\rangle /\left(q_{\mathrm{ref}}\right)^{2},\left\langle u^{\prime} v^{\prime}\right\rangle /\left(q_{\mathrm{ref}}\right)^{2}$, and $\left\langle v^{\prime} v^{\prime}\right\rangle /\left(q_{\mathrm{ref}}\right)^{2}$. Contour interval is 0.010 or 0.005
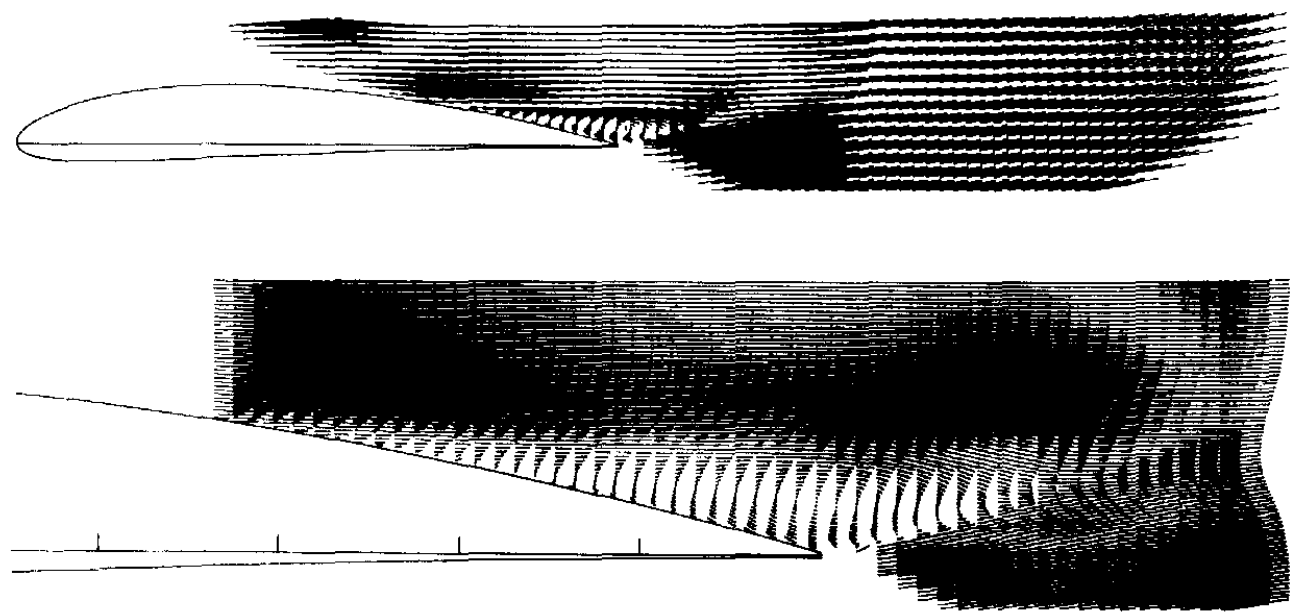

Fïg. 12 Partial display of vector field of mean velocity. Top: same scale as Figs. 10 and 11 ; bottom: close-up view of separated region. (Tick marks on chord line are at intervals of 0.1 in. $x / c$.) 
derstanding lies in the merging process for the two shear layers just downstream of the trailing edge and in the subsequent rapid relaxation toward the final state of a conventional wake far downstream.

Figure 12 is a partial display of mean-velocity vectors, first to the same scale as Figs. 10 and 11 and then as a close-up view of the separated region. The latter view shows quite graphically the evolution of the mean-velocity profile. There is some evidence in the figure of probe interference very close to the airfoil surface, where the first few vectors sometimes have an unnatural magnitude or an unnatural direction or both.

These final processed data have also been recorded on 29,195 punched cards and filed with the Large-Scale Aerodynamics Branch at NASA Ames Research Center.

\section{Discussion}

It is consistent with the purpose of the present research, as set out in the Introduction, that the data are presented here almost without interpretation. We assume that users of the data for purposes of turbulence modeling will eventually reach a concensus as to the strengths and weaknesses of the measurements, as did users of the earlier measurements by Schubauer and Klebanoff. ${ }^{2}$ We have made every effort, both here and in the parent reports $\mathrm{CW}$ and $\mathrm{CCW}$, to describe the experiment completely and to record both raw and processed data in accessible form. Because the passage from raw to processed data was not only tedious but expensive, we expect that most users will be willing to begin with the processed version of the data. However, we do not expect most users to agree quantitatively on derived quantities, such as stream function, or on the credibility of the data in terms of the Reynolds equations which govern the mean flow and the various moments of the fluctuating velocity field. Thus, for example, we have not published our estimates for friction on the airfoil surface. One reason is that we prefer to leave open the question of alternative coordinate systems for better describing the flow near the surface; another is that we doubt in any case that the surface friction in the separated region is very important for the dynamics of the motion.

Finally, we emphasize once more that the spirit of the present measurements is entirely the spirit of Reynolds averaging, with the additional feature that the boundary-layer approximation is avoided. We expect that for some time to come the measurements will provide a major test case for development of calculation methods for turbulent flow.

\section{Acknowledgment}

This research was supported by the Large-Scale Aerodynamics Branch, NASA Ames Research Center, Moffett Field, Calif., under Grants NGL 05-002-229 and NSG 2319.

\section{References}

${ }^{1}$ Coles, D.E. and Hirst, E.A. (Eds.) Computation of Turbulent Boundary Layers, Vol. II, Compiled Data, Proceedings of AFOSRIFP-Stanford Conference, Department of Mechanical Engineering, Stanford University, 1968.

${ }^{2}$ Schubauer, G.B. and Klebanoff, P.S., "Investigation of Separation of the Turbulent Boundary Layer," NACA TN 2133 , 1950.

${ }^{3}$ Simpson, R.L., Strickland, J.H., and Barr, P.W., "Features of a Separating Turbulent Boundary Layer in the Vicinity of Separation," Journal of Fluid Mechanics, Vol. 79, 1977, pp. 553-594.

${ }^{4}$ Seetharam, H.C. and Wentz Jr., W.H., "Experimental Investigation of Subsonic Turbulent Separated Boundary Layers on an Airfoil," Journal of Aircraft, Vol. 14, Jan. 1977, pp. 51-55.

s"CCW" Coles, D., Cantwell, B., and Wadcock, A., "The Flying Hot Wire and Related Instrumentation," Tech. Rept. to NASA Ames Research Center, NASA Grant NGL 05-002-229, 1977; also NASA Confr. Rep. CR-3066, 1978.

${ }^{6}$ Wadcock, A.J., "Flying-Hol-Wire Study of Two-Dimensional Turbulent Separation on an NACA 4412 Airfoil at Maximum Lift," $\mathrm{Ph}$.D. Thesis, California Institute of Technology, 1978.

7."CW" Coles, D. and Wadcock, A., "Flying-hot-wire study of Two-Dimensional Mean Flow past an NACA 4412 Airfoil at Maximum Lift," Tech. Rept. to NASA Ames Research Center, NASA Grants NGL 05-002-229 and NSG-2319, 1978.

${ }^{8}$ Cantwell, B.J., "A Flying Hot Wire Study of the Turbulent Near Wake of a Circular Cylinder at a Reynolds Number of 140,000," Ph.D. Thesis, California Institute of Technology, 1976.

9Pinkerton, R.M., "The Variation with Reynolds Number of Pressure Distribution Over an Airfoil Section," NACA Rept. 613, 1938.

${ }^{10}$ Perry, A.E. and Morrison, G.L., "A Study of the ConstantTemperature Hot-Wire Anemometer," Journal of Fluid Mechanics, Vol. 47, 1971, pp. 577-599.

"Abbott, I.H. and von Doenhoff, A.E., Theory of Wing Sections, McGraw-Hill, New York, 1949 (reprinted, Dover, 1959). 